\title{
Proposal of a quali-quantitative assessment model for health and safety in small and medium enterprises
}

\author{
M. Fera \& R. Macchiaroli \\ Department of Mechanical and Aerospace Engineering, \\ Second University of Naples, Italy
}

\begin{abstract}
The identification, assessment and reduction of risks is one of the most important elements of health and safety at work. Nowadays, safety at work is becoming an Italian and a globally relevant ethical and technical problem. Risk can be assessed with different methods: qualitative, quantitative or quali-quantitative; while qualitative models are often too simplistic, in turn many times quantitative models are difficult to implement in small and medium enterprises (SMEs). The aim of this work is to present a new practical assessment model that tries to overcome the mentioned difficulties. Beyond its description, the paper also proposes an application study made in an international firm production plant to validate its results.
\end{abstract}

Keywords: assessment methods, FMECA, SCEBRA, AHP.

\section{Introduction}

The injuries statistics (table 1) released by the International Labour Organization (ILO) for 2007 are awesome. They show very clearly how the health and safety problem is very far from a solution. Moreover, the analysis of the last two decades data reveals a general negative trend about injuries and deaths, caused by an inappropriate approach to health and safety at work. It is well known that an effective approach to health and safety at work requires a suitable risk assessment phase, the adoption of prevention and protection actions and the implementation of a severe inspection and control phase, or, in a wider sense, what is called a "safety audit". Instead, so far, these phases were faced in 
Table 1: $\quad$ Worldwide 2007 injuries and deaths.

\begin{tabular}{|l|l|}
\hline Type of injury & Number of injuries \\
\hline & $\begin{array}{l}250.000 .000 \mathrm{inj} / \mathrm{yr} \\
685.000 \mathrm{inj} / \text { day } \\
\text { Workplace Injury }\end{array}$ \\
& $8 \mathrm{inj} / \mathrm{sec}$ \\
\hline $\begin{array}{l}\text { Children Workplace } \\
\text { Injuries }\end{array}$ & $12.000 .000 \mathrm{inj} / \mathrm{yr}$ \\
\hline Deaths & $1.300 .000 \mathrm{death} / \mathrm{yr}$ \\
\hline
\end{tabular}

practice with non appropriate tools and methodologies, which are either too complex to manage or too simple and subjective, thus not suitable to recognize hazards and reduce the corresponding risks.

The aim of this paper is to present a new and reliable assessment model, which is able to face the aforesaid difficulties of the models developed so far. The proposed model is based on different techniques, such as the Failure Modes and Effects Criticality Analysis (FMECA), the Scenario Based Risk Assessment (SceBRA) and the Italian standard UNI 7249:2007. These techniques are merged through a procedure, composed of seven steps, some quantitative and some qualitative. This model also includes the decision making technique named Analytic Hierarchy Process (AHP), which - as well known - is useful to minimize inconsistencies in experts' judgements, within the qualitative phases of risk assessment.

\section{Literature review and open issues}

The identification and choice of a suitable risk assessment model has been felt to be a crucial issue for decades. So far, the models used in practice were developed for different applications and adapted for health and safety at work. A possible classification is presented in table 2 .

Table 2: $\quad$ Methods for the health and safety assessment.

\begin{tabular}{|c|c|}
\hline \multirow{3}{*}{ Qualitative } & "What if?" Analysis \\
\cline { 2 - 2 } & Safety review \\
\cline { 2 - 2 } Quantitative & Check lists \\
\cline { 2 - 2 } & Fault Tree Analysis \\
\cline { 2 - 2 } & Events Tree \\
\hline \multirow{2}{*}{$\begin{array}{c}\text { Quali- } \\
\text { quantitative }\end{array}$} & Bow-Tie model \\
\cline { 2 - 2 } & Fazards and Operability Study (HAZOP) \\
\hline
\end{tabular}

Qualitative methods are mainly focused on the experts' contribution. Experts are responsible for predicting the possible interactions between workers, machines and the work environment. Qualitative models cannot be implemented in all kinds of firms, because of their intrinsic content of uncertainty, which makes them unsuitable for several applications; for example, risk assessment in the chemical or oil and gas industries, where generally sophisticated reliability 
models can and must be applied, normally lead to a wide extent of success. Instead, they can be used with good results in non industrial environments. In the international literature there are some contributions about risk assessment for fishing vessels [1], using the check-lists method, or for large transport networks and urban systems [2]. Other authors [3] developed a qualitative assessment model based on the European guidelines RAPEX, used for food quality and safety assurance. These models are often used in small and medium enterprises (SMEs) due to their simplicity, though their application can be misleading, as underlined by many authors [4-6].

On the other hand, quantitative models are widely used in many fields, such as in large industrial firms or in the petrochemical industry. These models make extensive use of reliability analysis and, thus, are based on process decomposition techniques and failures probability knowledge. Indeed, several works appearing in the literature are based on the Bayesian approach for fault tree analysis or for event trees analysis $[7,8]$. The statistic approach is also used for other types of quantitative risk assessment models, as for the Bow-Tie model [9]. Bow-Tie models are based on the identification of a link between causes and effects of events, and create a direct quantitative relation between risk sources and risk consequences. All the possible paths from the cause to the effect are associated to a likelihood that is the expression of the relative importance of a specific risk as connected to a risk source. Quantitative methods are used to assess risks in the chemical field $[10,11]$ or in the coal mines [12]; in these sectors safety is often related to specific possible accidents, whose severity justifies the adoption of quantitative evaluation techniques.

The existing literature does not report many works using mixed qualiquantitative methods. Some apply typical techniques of knowledge analysis, such as fuzzy theory [13], to try to formalize and quantify subjective aspects, treated as fuzzy variables.

The analysis of the existing literature convinced us that there is space for work on quali-quantitative methods. The lack of such approaches can be due, in our opinion, to the low attention paid to safety in SMEs by researchers and practitioners. This fact, in turn, might be due to the higher interest paid to larger industrial firms, which - in a first analysis - could be identified as a major risk source. Since all statistics show, instead, that most injuries and deaths are more likely to occur in SMEs, the purpose of this work is to propose a qualiquantitative risk assessment method, which is able to overcome the practical difficulties generally found by SMEs in the application of quantitative techniques (also due to the high skills required for this aim) and to fill the gap between the results obtained by the application of a qualitative approach, generally employed by SMEs, and the need for a reliable risk assessment.

\section{The proposal}

As anticipated in the previous sections of this paper, our proposal makes use of and merges different techniques. 
The model is organized into three main phases, and each phase is composed of different steps that involve methods, such as FMECA, SCEBRA, the Italian standard UNI 7249:2007 and AHP. Each step makes use of different methodologies. Table 3 reports the methods used for each step.

The model integrates a typical technique used in maintenance design (FMECA) with a risk assessment management technique (SceBRA) and with the Italian standard UNI 7249:2007 used to evaluate risks frequencies and consequences. AHP is used in steps 2 and 6 to minimize the subjectivity of experts' contributions. Figure 1 reports the sequence of the three phases in the proposed model.

In the first phase the work team is built and major risks are identified; this phase makes use of the SceBRA and AHP techniques. The second phase of the assessment procedure is focused on the risk criticality calculation, performed using the Italian standard and the FMECA technique. In the last phase preventive and protective actions to minimize the risks are identified and classified according to their priority; using the AHP. Step 7 also includes verification and validation of the improvements achieved.

Table 3: $\quad$ Methods used for each step.

\begin{tabular}{|c|l|l|}
\hline Step & \multicolumn{1}{|c|}{ Description } & \multicolumn{1}{c|}{ Method } \\
\hline 1 & Team work identification & SceBRA \\
\hline 2 & Major events identification & SceBRA-AHP \\
\hline 3 & Frequencies calculation & FMECA-UNI 7249 \\
\hline 4 & Consequences calculation & FMECA-UNI 7249 \\
\hline 5 & Criticality calculation actions & FMECA \\
\hline 6 & $\begin{array}{l}\text { Improvement } \\
\text { prioritization }\end{array}$ & Mixed techniques \\
\hline 7 & Improvement action validation & \\
\hline
\end{tabular}

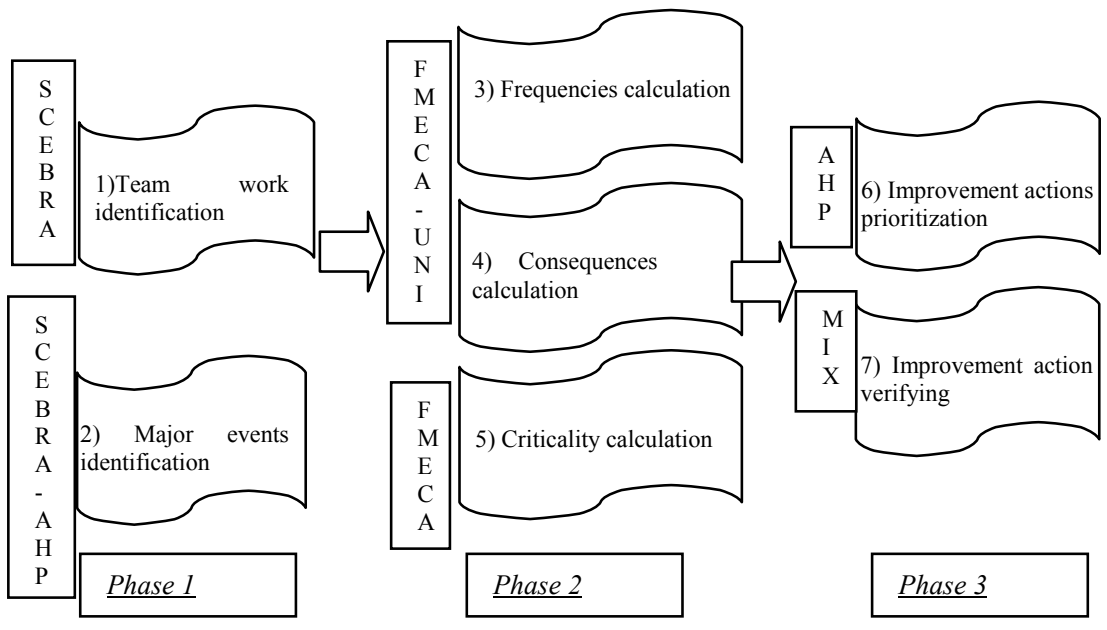

Figure 1: Assessment model. 
The SceBRA risk assessment technique is widely used in financial management, especially when one needs to analyse and forecast different scenarios. This technique has seldom been used for safety risks analysis. In our model, having recognized that a typical critical issue in risk assessment is often its identification, we employed the first part of the SceBRA technique which is focused on the construction of an effective work team.

In the literature it is possible to find out some contributions that use the FMECA to assess safety problems. Indeed, FMECA is used in our model to calculate risks criticality.

UNI 7249:2007 is an Italian standard that illustrates calculation methods for the hazards occurrence probability and consequence indexes, starting from the injuries data available in each firm.

AHP has been selected to reduce the subjectivity of the steps 2 and 6 . AHP permits to give judgements of relative relevance between different risks, not just using numerical values, but also with verbal values (indeed, it includes a correspondence table from the verbal to numerical values). A comparative relevance judgement is given to each possible couple of risks by a pool of experts. All their judgement values are collected in a geometrical mean for each couple of risks. AHP uses the geometrical mean since it's possible to prove that if the judgements of different experts are collected through it, the corresponding judgements super matrix inconsistency is minimum [14]. Thus, the use of this technique achieves higher reliability levels.

Let us now describe in further details the steps the procedure is composed of.

First step, i.e., team building, is very important, because to ensure a reliable risk assessment it's worth to involve not only experts but also the personnel working in the environment under study. The team members can be gathered from the firm safety organization which, in turn, is set mainly by European laws. Since our will is to build an assessment model also able to comply with the safety regulations in force, a minimal possible composition of the team is the following: (i) the safety responsible, (ii) an occupational health expert and (iii) an expert of the working environment.

Second step, i.e., risk identification, is carried out with applying the AHP technique. Each expert gives a judgement of relative importance for each couple of risks, i.e., the expert has to say how much the analysed risk is relevant compared with all the others. The experts' relative importance judgement for each risks couple is synthesized using their geometrical mean. The final comparative judgement, i.e., the aforementioned means, is collected into a matrix used to understand the relative priorities of risks. The hierarchy used in the risks prioritization is reported in figure 2 .

Once the risks' priorities are obtained, it is possible to proceed to the third and fourth steps. In these two steps the model proposes the calculation of the frequency and consequence indexes of hazards. These two indexes are calculated according to Italian national standard, UNI 7249:2007 (this standard is available also in English as "Statistics on occupational injuries"), which proposes equations (1) and (2). 

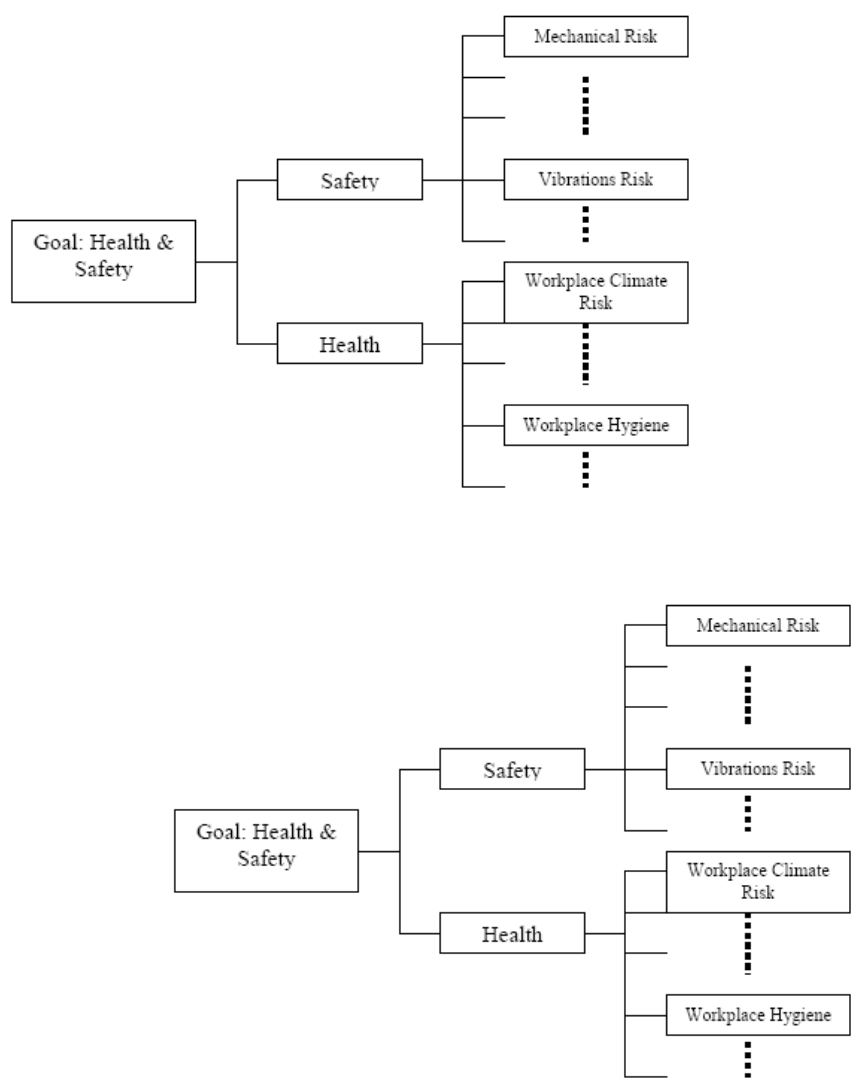

Figure 2: $\quad$ Risk hierarchy.

$$
\begin{gathered}
F=\frac{{ }_{I} A+{ }_{I} m}{E_{h}} \cdot 10^{6} \\
C=\frac{{ }_{I} G_{T}+{ }_{I} G_{P}+{ }_{I} G_{M}}{E} \cdot 10^{3}=\frac{{ }_{I} G_{T}+7500 \cdot\left(\frac{\sum g}{100}\right)+7500 \cdot m}{E} \cdot 10^{3}
\end{gathered}
$$

In (1), ${ }_{\mathrm{I}} \mathrm{A}$ is the number of injuries causing a leave greater than a day, ${ }_{\mathrm{I}} \mathrm{m}$ is the number of deaths and $E_{h}$ is the worked hours. In (2) ${ }_{I} G_{T},{ }_{I} G_{P},{ }_{I} G_{M}$ are respectively the working absence days due to (i) a temporary injury, (ii) to an injury with permanent consequences and (iii) to a death, and E is (iv) the total number of workers employed in the specific work environment. All these data are normally available in the firm's injury registration book. 
Afterwards, the procedure starts to implement the fifth step, i.e., the general criticality index calculation that collects all the previous results. The relation used to calculate it is reported in (3).

$$
I_{k}=F_{k} \cdot C_{k}+p_{k} \cdot \Delta(F \cdot C)=F_{k} \cdot C_{k}+p_{k} \cdot\left[(F \cdot C)_{\max }-(F \cdot C)_{\min }\right]
$$

where $F$ and $C$ are, respectively, the frequency and the consequence indexes for the $k$-th risk analysed, $p_{k}$ is the priority index calculated as in step 2 and $\Delta(F C)$ is the difference between the maximum and the minimum value of all the risks criticalities as computed in the first term. The second term was introduced to assess all risks with no history, i.e., for the ones where it's not possible to compute frequency and consequences indexes. Moreover, it is important to note that the determination of the second term is strictly related to the environment analysed, because it depends, one on hand, on the risk relevance (through $p_{k}$ ), calculated with the judgements of the experts analysing the firm environment, and, on the other, on a measure of the general risk level of the firm (through $\Delta(F C)$ ); both these values are typical of a specific firm and they cannot be used in other firms.

The sixth step consists in. the identification of prevention and protection measures, respectively, to reduce frequencies and consequences of hazards. This step makes use again of AHP by the persons defined in the step 1. For each risk, the experts will define some actions, and afterwards they will express their relative judgements between the proposed actions. The calculation procedure to obtain the priorities indexes is the same of step 2. The improving actions hierarchy is shown in figure 3 .

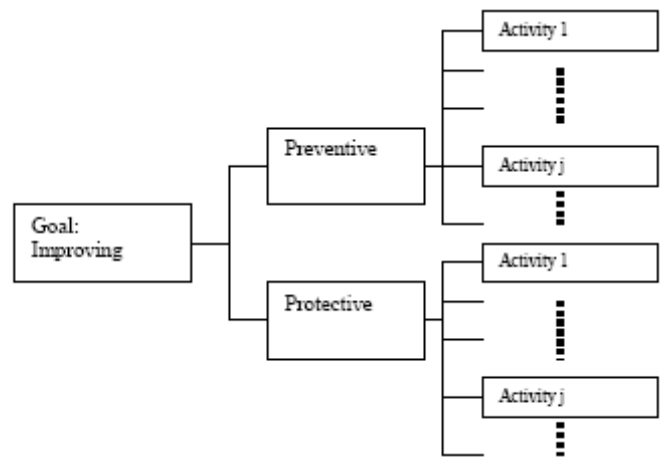

Figure 3: Improving action hierarchy.

Once the actions just defined are actually implemented, there's a need to validate their effectiveness. This is performed by the seventh step. In this proposal it is described the use of an index, named DOE, created by the US Department of Energy. The DOE index formula is reported in (4).

$$
D O E=\frac{10^{2} \cdot\left(10^{6} \cdot D+5 \cdot 10^{5} \cdot T+2 \cdot 10^{3} \cdot L W C+10^{3} \cdot W D L+4 \cdot 10^{2} \cdot W D L R+2 \cdot 10^{3} \cdot N F C\right)}{E_{h}}
$$


In (4) $\mathrm{D}$ is the number of deaths, $\mathrm{T}$ is the number of injuries with total disability, LWC is the number of accidents with an injury, WDL is the number of days of absence from work, WDLR is the number of days in which a production area had to work in a downsized mode, NFC is the number of near miss and $E_{h}$ is the total number of working hours. Using this index over an appropriate time horizon, it is possible to calculate which safety system results in improvement. If the index trend passing from a period to another shows a negative result, i.e., if the difference of the indexes, as computed in two periods, is positive or equal to zero, it means that the assessment and, so, the improved actions were not adequate, so another implementation of the procedure described so far is needed.

\section{The validation}

The validation of the model was performed through an experimental campaign carried out in an Italian SME. The analysed risks are related to a specific production line, shown in figure 4. It is an assembly line, operating on a 24 hours basis. Activities carried out by workers include control tasks, feeding of raw material feed to machinery and machines set-up for each cycle. Production makes use of chemical products, such as flammable or noxious products.

All risks were identified and assessed using the proposed model. Criticality indexes, computed according to the methodology, are reported in table 4.

Table 5 reports the results of the proposed method, of the old assessment method based on the experts' knowledge and the real classification of the risks. The risks' real classification is drawn by the injuries reported in the firm's registration book, multiplying the frequency and consequences indexes for each

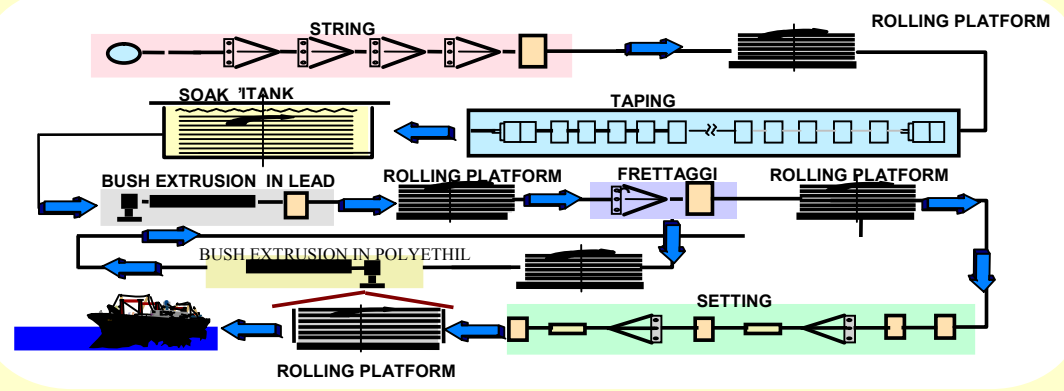

Figure 4: Assembly line analysed. 
Table 4: $\quad$ Criticality index for the risks in the assembly line analysed.

\begin{tabular}{|r|c|c|c|}
\hline \multicolumn{1}{|c|}{ Risk } & $\mathrm{F}^{* \mathrm{C}}$ & $\mathrm{p}_{\mathrm{k}} * \Delta(\mathrm{F} \mathrm{C})$ & $\mathrm{I}_{\mathrm{k}}$ \\
\hline Mechanical & 26,49 & 7,15 & 33,64 \\
\hline Noise & 0,00 & 4,24 & 4,24 \\
\hline Knife parts contact & 6,14 & 4,00 & 10,14 \\
\hline Material in movement & 4,04 & 2,65 & 6,69 \\
contact & & & 6,38 \\
\hline Electric & 3,99 & 2,39 & 1,32 \\
\hline Fire & 0,00 & 1,32 & 1,11 \\
\hline Vibrations & 0,32 & 0,79 & \\
\hline
\end{tabular}

Table 5: Comparison among the classification methods and the real data.

\begin{tabular}{|c|c|c|}
\hline New assessment method & Real data & Old assessment method \\
\hline Mechanical & Mechanical & Noise \\
\hline Knife parts contact & Knife parts contact & Mechanical \\
\hline Material in movement contact & Material in movement contact & Electric \\
\hline Electric & Electric & Vibrations \\
\hline Noise & Vibrations & Material in movement contacts \\
\hline Fire & Fire & Climate \\
\hline Vibrations & Noise & Chemical \\
\hline Chemical & Chemical & \\
\hline
\end{tabular}

Table 6: $\quad$ Methods reliability.

\begin{tabular}{|c|c|c|}
\hline & New assessment method & Old assessment method \\
\hline Reliability & $75 \%$ & $37.5 \%$ \\
\hline
\end{tabular}

risk. The comparison of the times in which the methods reflect the same results shown by the real risk classification, makes it possible to understand the reliability of each method. The comparison just described is reported in table 5 .

The new model matches the real risk classification more often than the old assessment method. In table 6, the matching scores of the proposed and the old methods are reported.

From the data reported in table 6 it is possible to infer that the new method proposed achieves a better level of reliability.

In conclusion, based on the results of the application of the proposed methodology to the chosen test bench, it's possible to state that the new model proposed in this paper represents an improvement for the safety assessment reliability. In particular, the new method presents a "matching score" with real results that is the double of the old evaluation method's one. So, after this first experimental campaign, the results obtained are encouraging and stimulating us to continue with the experimentation of this new method, that will be applied in different workplaces with different sizes.

\section{References}

[1] Piniella et al., Towards system for the management of safety on board artisanal fishing vessels: Proposal for check-lists and their application, Safety Science 47 (2009) p. 265-276 
[2] Chen et al., Study on the methodology for evaluating urban and regional disaster carrying capacity and its application, Safety Science, Volume 47, Issue 1, (2009), p. 50-58

[3] Van Duijne et al., Considerations in developing complete and quantified methods for risk assessment, Safety Science 46 (2008), p. 245-254

[4] Hetherington et al., Safety in shipping: The human element, Journal of Safety Research 37 (2006), p. 401-411

[5] Wirth et al., When workplace safety depends on behaviour change: Topics for behavioural safety research, Journal of Safety Research 39 (2008) p. 589-598

[6] Lingard et al., Behaviour-Based in Hong Kong's Safety Management Construction Industry, Journal of Safety Research. Vol. 28 No. 4. (1997) p. 243-256

[7] Martìn et al., A Bayesian network analysis of workplace accidents caused by falls from a height, Safety Science 47 (2009) p. 206-214

[8] Doytchev et al., Combining task analysis and fault tree analysis for accident and incident analysis: A case study from Bulgaria, Accid. Anal. Prev. (2008), doi:10.1016/j.aap.2008.07.014

[9] Ale et al., Quantifying occupational risk: The development of an occupational risk model, Safety Science 46 (2008), p. 176-185

[10] Glickman et al., Assessment of hazardous material risks for rail yard safety, Safety Science 45 (2007), p. 813-822

[11] Brito et al., Multi-attribute risk assessment for risk ranking of natural gas pipelines, Reliability Engineering and System Safety 94 (2009) p. 187-198

[12] Sari et al., Stochastic modelling of accident risks associated with an underground coal mine in Turkey, Safety Science 47 (2009), p. 78-87

[13] Grassi et al., A fuzzy multi-attribute model for risk evaluation in workplaces, Safety Sci. (2008), doi:10.1016/j.ssci.2008.10.002

[14] Saaty, T. L. (2000). Fundamentals of Decision Making and Priority Theory with the Analytic Hierarchy Process. Pittsburgh, PA: RWS Publications 